\title{
Adjustability of valves for shunting hydrocephalus is luxury, progress or necessity? Our personal experience to stimulate debate Christian Sprung* and Hans-Georg Schlosser
}

\author{
Address: Neurosurgical Clinic, Charité, Universitätsmedizin Berlin, Augustenburger Platz 1, 13353 Berlin, Germany \\ Email: Christian Sprung* - christian.sprung@ charite.de \\ * Corresponding author
}

from 52nd Annual Meeting of the Society for Research into Hydrocephalus and Spina Bifida

Providence, RI, USA. I I-14 June 2008

Published: 3 February 2009

Cerebrospinal Fluid Research 2009, 6(SuppI I):S45 doi:I0.II86/1743-8454-6-SI-S45

This abstract is available from: http://www.cerebrospinalfluidresearch.com/content/6/SI/S45

(c) 2009 Sprung and Schlosser; licensee BioMed Central Ltd.

\section{Background}

The obvious theoretical advantage of adjustable valves compared to those with fixed opening pressure could not be proven in the vast majority of published series especially in children. But this seems contradictory to the increasing sales numbers of so-called "programmable" valves speaking for an augmentation of acceptance. The reasons for the difficulty to prove the superiority of the latter are multiple and by our experience with a new adjustable valve we would like to elucidate this matter of controversy.

\section{Materials and methods}

The investigation was conducted by comparing our experience in valves with fixed opening-pressure and the evaluation of the results after implantation of the adjustable gravitation-assisted proGAV in 108 patients from 2/2004 to $4 / 2007$. We differentiated complications related to the valve-function from those not dependent on the device, and focused on indications to change the opening pressure. Finally we concentrated on the radiological and clinical results including the possibilities for improvement by readjustment.

\section{Results}

In 65 patients we did not see an indication to change the initial pressure setting and the clinical results were satisfying in 54. The combination of unsatisfying clinical outcome and unchanged ventricles necessitates to rule out shunt-insufficiency before adding this cohort to the group of non-responders. In 31 cases with the "complication" of functional underdrainage we saw an indication to lower the opening pressure with 29 responders. All 8 patients suffering overdrainage-related "complications" could be treated successfully by elevating the pressure-setting avoiding re-operations. 9 patients demonstrated signs of under - as well as overdrainage during follow-up.

\section{Conclusion}

The difficulties to prove superiority of adjustability stem not only from different inclusion criteria, variance in definition of complications or the variability in the choice of pressure setting in conventional D-P-valves, but are also due to the fact that too many patients were classified as belonging to the non-responders. Only those patients should be subsumed under the category of "non-responders", who do not improve clinically despite reduction of ventricles, change of valve or following adequate alteration of pressure setting in programmable devices. The results of the proGAV-series support our opinion that by adjustability many re-operations can be avoided and the amount of non-responders can be diminished. 